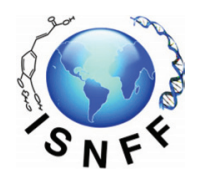

\title{
Lipophilized resveratrol affects the generation of reactive nitrogen species in murine macrophages and cell viability of human cancer cell lines
}

\author{
Won Young $\mathrm{Oh}^{\mathrm{a}}$, Yi-Shiou Chiou ${ }^{\mathrm{b}}$, Pei-Sheng Lee ${ }^{\mathrm{b}}$, Min-Hsiung Pan ${ }^{\mathrm{b}}$ \\ and Fereidoon Shahidi ${ }^{\mathrm{a}^{*}}$
}

\begin{abstract}
aDepartment of Biochemistry, Memorial University of Newfoundland, St. John's, NL, Canada A1B 3X9
bInstitute of Food Science \& Technology, National Taiwan University, Taipei, Taiwan 10617

${ }^{*}$ Corresponding author: Min-Hsiung, Institute of Food Science \& Technology, National Taiwan University, Taipei, Taiwan 10617. Tel: +886-2-3366-4133 E-mail: mhpan@ntu.edu.tw; Fereidoon Shahidi, Department of Biochemistry, Memorial University of Newfoundland, St. John's, NL, Canada A1B 3X9. Tel: +1 7098648552 E-mail: fshahidi@mun.ca

DOI: $10.31665 / J F B .2019 .7201$

Received: September 06, 2019; Revised received \& accepted: September 28, 2019

Citation: Oh, W.Y., Chiou, Y.-S., Lee, P.S., Pan, M.-H., and Shahidi, F. (2019). Lipophilized resveratrol affects the generation of reactive nitrogen species in murine macrophages and cell viability of human cancer cell lines. J. Food Bioact. 7: 73-77.
\end{abstract}

\begin{abstract}
Resveratrol was esterified with selected fatty acids to improve its lipophilicity and potential application in food and biological systems. In this study, resveratrol and monoesters of resveratryl propionate (RC3:0) and resveratryl docosahexaenate (RDHA) were examined for their effects on anti-inflammatory and anti-proliferative activity in vitro. All test compounds showed a decreased nitrite production in murine RAW 264.7 cells in a concentration dependent manner. Resveratrol, RC3:0, and RDHA were evaluated for their effects on cell viability using liver cancer (HepG2), colon cancer (HT-29, A431), breast cancer (MCF7), and gastric cancer (AGS) cell lines. All test compounds showed decreased cell viability of HepG2, A431, MCF7, HT-29, and AGS in a concentrationdependent manner. The results suggest that resveratrol esters may serve as potential anti-inflammatory and anti-proliferative agents.
\end{abstract}

Keywords: Resveratrol ester; Antioxidant activity; Anti-inflammatory activity; Anti-proliferative effect.

\section{Introduction}

Inflammation, such as redness, warmth, and swelling, is a normal reaction to injuries or infections. However, prolonged inflammation can progress further to various chronic diseases such as cardiovascular disease and cancer (Zhong et al., 2012). Reactive oxygen species (ROS) and reactive nitrogen species (RNS) may explain the link between inflammation and other chronic diseases (Wiseman and Halliwell, 1996). It is well known that ROS/RNS are continuously generated in vivo as a by-product of biological reactions (Halliwell, 1996, Fransen et al., 2012). Superoxide $\left(\mathrm{O}_{2}{ }^{-}\right)$, hydrogen peroxide $\left(\mathrm{H}_{2} \mathrm{O}_{2}\right)$, and nitric oxide $(\mathrm{ON} \bullet)$ are the primary ROS/RNS in a cell and they can readily form other ROS/RNS (Fransen et al., 2012). At a low level, ROS/RNS can serve as mediators for various biological responses such as gene expression and cell proliferation. However, at high levels, they can also cause harmful effects, including inflammation, cardiovascular disease, and cancer (Fransen et al., 2012; Zhong et al., 2012). Antioxidative defense systems in the body are frequently insufficient to keep ROS/RNS level in balance, therefore external sources of antioxidants are needed for this purpose (Yu, 1994; Loft and Poulsen, 1996).

Resveratrol is a powerful antioxidant and has been found in more than 70 plant species, especially grape skins. Red wine also contains resveratrol that originates from grapes (Dercks and Creasy, 1989). Resveratrol has received increasing attention since red wine was first shown to display cardioprotective effects (Baur and Sinclair, 2006). In addition, resveratrol prevents oxidation, cancer, coronary heart disease and inflammation (Jang et al., 1997; Wang et 
al., 2002; Donnelly et al., 2004). Although it has beneficial health properties, there are limitations to its bioactivity due to its hydrophilic nature and fast metabolism in the body (Zhong and Shahidi, 2011; Walle et al., 2004). Therefore, structural modification may provide an opportunity to address this concern (Torres et al., 2010; Baur and Sinclair, 2006). Several studies on lipophilic derivatives of phenolic compounds, including resveratrol, have demonstrated their better performance in biological model systems. Acetylated resveratrol showed better inhibition of platelet activating factor (PAF; 1-O-alkyl-2-acetyl-sn-glycero-3-phosphocholine)-induced washed rabbit platelet aggregation and DU-145 human prostate cancer cell-growth than resveratrol itself (Fragopoulou et al., 2007; Cardile et al., 2005). In addition, Cichocki et al. (2008) reported that pterostilbene (dimethylated resveratrol) acted as an inhibitor of tumour promotion biomarker. In addition, Pan et al. (2007) reported that pterostilbene was able to inhibit cell proliferation and induce apoptosis in human gastric carcinoma cells. Moreover, esterification of resveratrol with polyunsaturated fatty acids (PUFAs) may provide a synergistic effect due to the individual bioactivities of its components. In addition, our preliminary data showed that some of the resveratrol esters had an effect on RNS generation in murine macrophages. For this reason, resveratrol was esterified with propionyl chloride (C3:0) and docosahexaenoyl chloride (DHA, C22:6), as described elsewhere (Oh and Shahidi, 2017). In this study, the mixture of R-3-O- and R-4'-O-monoesters (RC3:0 and RDHA) were evaluated for inhibition against $\mathrm{NO}$ production as well as antiproliferative effect in various cancer cell lines.

\section{Materials and methods}

\subsection{Materials}

Resveratrol and DHA were acquired from DSM Nutritional Products (Columbia, MD, USA). The RAW 264.7 cells, derived from murine macrophages, were procured from the American Type Culture Collection (Rockville, MD, USA). Heat-inactivated fetal calf serum was purchased from GIBCO (Grand Island, NY, USA). Lipopolysaccharide (LPS; Escherichia coli O127:E8, molecular weight, $60 \mathrm{kDa}$ ) was purchased from Sigma Chemical Co. (St. Louis, MO, USA). Human hepatocellular carcinoma (HepG2) cells (BCRC 60025) were procured from the Food Industry Research and Development Institute (Hsinchu, Taiwan). The human A431, AGS, and MCF7 cell lines were isolated from epidermoid carcinoma (ATCC CRL-1555), gastric adenocarcinoma (ATCC CRL-1739) and breast adenocarcinoma (ATCC HTB-22), respectively. Fetal bovine serum (FBS) was bought from Biological Industries (Cromwell, CT, USA).

\subsection{Preparation of resveratrol esters}

Preparation of resveratrol esters was the same as that previously described (Oh and Shahidi, 2017). Resveratrol was lipophilized with propionyl chloride and docosahexaenoyl chloride. The monoesters were identified by HPLC-MS and several types of NMR $\left({ }^{1} \mathrm{H}\right.$ NMR, ${ }^{13} \mathrm{C}$ NMR, COSY, HSQC, NOESY, and HMBC). Resveratrol and its esters (Figure 1) were subjected to NO production and cell viability assay evaluations.

\subsection{Cell culture}

The RAW 264.7 cells, derived from murine macrophages, were<smiles>[R]Oc1cc(O)cc(/C=C/c2ccc(O)cc2)c1</smiles><smiles>[R]Oc1ccc(/C=C/c2cc(O)cc(O)c2)cc1</smiles>

$\mathrm{R}: \mathrm{C}_{3} \mathrm{H}_{6} \mathrm{O}(\mathrm{C} 3: 0)$

$$
\mathrm{C}_{22} \mathrm{H}_{32} \mathrm{O}(\mathrm{C} 22: 6 \mathrm{n}-3 \text {; DHA })
$$

Figure 1. Structures of RC3:0 and RDHA monoesters.

cultured in Dulbecco's minimal essential medium (DMEM) containing $10 \%$ endotoxin-free, heat-inactivated fetal calf serum, 100 units $/ \mathrm{mL}$ penicillin, and $100 \mu \mathrm{g} / \mathrm{mL}$ streptomycin. The AGS cell lines in DMEM-F12 were grown at $37{ }^{\circ} \mathrm{C}$ in $5 \% \mathrm{CO}_{2}$ atmosphere. HepG2, A431, and MCF7 cell lines were grown in DMEM supplemented with $10 \%$ heat-inactivated fetal calf serum, 100 units/ $\mathrm{mL}$ of penicillin, and $100 \mu \mathrm{g} / \mathrm{mL}$ of streptomycin. Those cell lines were kept at $37^{\circ} \mathrm{C}$ in a humidified atmosphere of $5 \% \mathrm{CO}_{2}$.

\subsection{Nitrite assay}

The cells were seeded into 24 well plates, after reaching a density of $1 \times 10^{6}$ cells $/ \mathrm{mL}$ and were activated by changing their medium to serum-free DMEM without phenol red containing LPS (Zhong et al., 2012). The RAW 264.7 cells were treated with different concentrations of each compound and LPS or LPS alone for 24 h. The supernatants $(100 \mu \mathrm{L})$ were mixed with the Griess reagent (100 $\mu \mathrm{L}, 1 \%$ sulphanilamide in $5 \%$ phosphoric acid and $0.1 \%$ naphthylethylenediamine dihydrochloride in water) in duplicate in 96-well plates. The absorbance was then read at $570 \mathrm{~nm}$ with an ELISA reader. Sodium nitrite $\left(\mathrm{NaNO}_{2}\right)$ was employed as a standard.

\subsection{Cell viability of HepG2, A431, MCF7, HT-29, and AGS}

Resveratrol and its esters were dissolved in dimethyl sulfoxide (DMSO). Cell viability of various cancer cell lines was assessed by using 3-(4,5-dimethylthiazol-2-yl)-2,5-diphenyltetrazoliumbromide (MTT) assay (Mosmann, 1983). The cells were plated at a density of $2 \times 10^{5}$ cells $/ \mathrm{mL}$ into 96 well plates and incubated for 


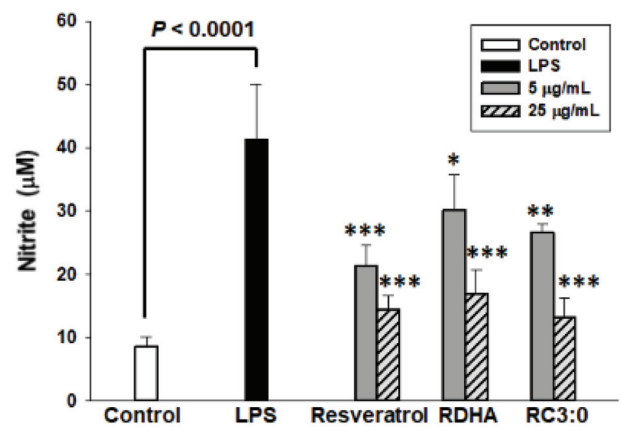

Figure 2. Effect of resveratrol and its monoesters on NO production in LPS-induced RAW 264.7 macrophages. RAW264.7 cells were treated with resveratrol, RDHA and RC3:0 different concentrations and LPS $(100 \mathrm{ng} / \mathrm{mL})$ for $24 \mathrm{~h}$. Results were statistically analysed with one-way ANOVA followed by Tukey's post hoc test. Statistical significance, ${ }^{*} P<0.05,{ }^{* *} P<0.01$ and $* * * P<0.001$, compared with the LPS-treated group

$24 \mathrm{~h}$. The cells were then pretreated with test compounds at different concentration for $24 \mathrm{~h}$. The final concentration of DMSO in the culture medium was $<0.05 \%(\mathrm{v} / \mathrm{v})$. MTT $(100 \mu \mathrm{L})$ was added, and the cells were incubated for a further $1 \mathrm{~h}$. Then the medium was aspirated and $100 \mu \mathrm{L}$ of DMSO were added to dissolve the MTT-formazan crystals formed by metabolically viable cells. Cell viability was scanned with an enzyme-linked immunosorbent assay reader with a $570 \mathrm{~nm}$ filter.

\section{Results and discussions}

\subsection{Nitric oxide (NO) production}

NO is an important molecule with known beneficial activities such as vascular relaxation and neurotransmission in mammals (Coleman, 2001). The solubility and diffusion properties of NO are similar to those of oxygen and it can freely cross cell membranes and has a long half-life (Coleman, 2001). It is a relatively stable radical, however it can form peroxynitrite anion $\left(\mathrm{ONOO}^{-}\right)$which is a more reactive form of RNS (Kruidenier and Verspaget, 2002). Moreover, presence of an excessive amount of NO in activated immune cells leads to tissue damage during inflammation (Zhong et al., 2012). NO is generally synthesized from L-arginine by three different types of NO synthase (NOS), namely neural NO synthase (nNOS), endothelium NO synthase (eNOS), and inducible NO synthase (iNOS). Unlike nNOS and eNOS, iNOS is continuously active once expressed and produces $\mathrm{NO}$ at a sustained high level. Expression of iNOS can be induced by LPS or cytokines such as tumour necrosis factor (TNF)- $\alpha$ and interleukin (IL)-1 (Coleman, 2001).

In this study, inhibition of LPS-induced NO production in murine RAW 264.7 was examined for two different resveratrol esters (Figure 1) and compared with resveratrol. The nitrite concentration in the culture medium was considered as NO production. As shown in Figure 2, the nitrite level of LPS treatment alone (LPS) increased greatly compared to that of the control $(p<0.005)$. All samples were able to attenuate NO production in murine RAW 264.7 cells. Resveratrol and RC3:0 showed decrease of NO production at both 5 and $25 \mu \mathrm{g} / \mathrm{mL}$. However, RDHA at $5 \mu \mathrm{g} / \mathrm{mL}$ was able to attenuate the NO production slightly but an insignificant difference was observed as compared to LPS; RDHA at $25 \mu \mathrm{g} / \mathrm{mL}$ was able to inhibit overproduction of NO effectively and it was as effective as resveratrol itself at the same concentration.

Tsai et al. (1999) reported that resveratrol inhibited the generation of NO and reduced the level of iNOS protein. They also found almost complete suppression of iNOS mRNA by resveratrol, suggesting that the inhibition of iNOS generation might be due to suppression of iNOS mRNA. Bi et al. (2005) reported that resveratrol inhibited the release of NO as well as TNF- $\alpha$ induced by LPS. Zhong et al. (1999) stated that resveratrol showed inhibition of IL- 6 release. TNF- $\alpha$ and IL- 6 are involved in proinflammatory responses. Resveratrol esters have not been studied on the iNOS protein generation, iNOS gene expression, and the release of IL- 6 and TNF- $\alpha$, however they are expected to exert an effect on pro-inflammatory agents due to possessing inhibitory activity on NO production. This implies that all tested compounds may render an inhibitory effect on NO production and possibly serve as anti-inflammatory agents, but this needs to be further investigated.

\subsection{Effect of resveratrol and its esters on the viability of HepG2, A431, MCF7, HT-29, and AGS cells}

The effect of resveratrol and its esters at various concentrations on the viability of HepG2, A431, MCF7, HT-29, and AGS cells was determined by employing the MTT assay. As shown in Figure 3, resveratrol and its esters decreased the growth in cultured human cancer cells. Resveratrol at $5 \mu \mathrm{g} / \mathrm{mL}$ was able to decrease cell viability in all tested cancer cell lines except for A431 cells, whereas $5 \mu \mathrm{g} / \mathrm{mL}$ of RC3:0 showed cytotoxicity in all cancer cells. Concentration at $50-75 \mu \mathrm{g} / \mathrm{mL}$, resveratrol decreased the cell viability less than 40\% in HepG2, MCF7, HT29, and AGS, whereas RC3:0 showed less than $40 \%$ cell viability in HepG2, A431, MCF7, and AGS. RDHA at all concentrations showed the lowest cytotoxicity in HePG2, A431, HT29, and AGS cells compared to resveratrol and RC3:0. However, 50 and $75 \mu \mathrm{g} / \mathrm{mL}$ of RDHA in MCF7 cells showed no cell viability.

Several studies have demonstrated the anticancer activity of resveratrol and its derivatives. Jang et al. (1997) studied the anticancer activity of resveratrol in three major stages of carcinogenesis and found its chemopreventive effect due to acting as an antioxidant, antimutagen, anti-inflammatory, antipromotion, and antiprogression agent. In addition, resveratrol inhibited preneoplastic lesions in a mouse mammary glands culture model of carcinogenesis and inhibited tumorigenesis in a mouse skin cancer model. Pan et al. (2007) demonstrated that pterostilbene also possessed effects on cancer cells. They studied the inhibition of cell proliferation in pterostilbene treated human cancer cells such as COLO 205, AGS, HL-60, HepG2, and HT-29. They reported that pterostilbene $(60 \mu \mathrm{M})$ showed the largest sensitive inhibitory effect on AGS cell-growth compared to the other cell tested. Patel et al. (2010) revealed the concentration of resveratrol and its metabolites in the colorectal tissue of cancer patients and the potential benefit of resveratrol as a chemopreventive agent in colorectal cancer. They found that daily doses of 0.5 or $1.0 \mathrm{~g}$ resveratrol could reduce cancer cell proliferation by $5 \%$. However, some studies have failed to show such anticancer activity. For example, Bove et al. (2002) found that resveratrol showed inhibitory effect on the growth of 4T1 breast cancer cell line (in vitro) in a doseand time-dependent manner, however it showed no effect on the growth of 4T1 breast cancer in mice (in vivo). Baur and Sinclair (2006) suggested that this failure could be due to an inadequate dose of resveratrol, delivery method, and tumor origin. In pharmacokinetic perspective, resveratrol has extremely low bioavailability, and increasing the dose of resveratrol to overcome its low 

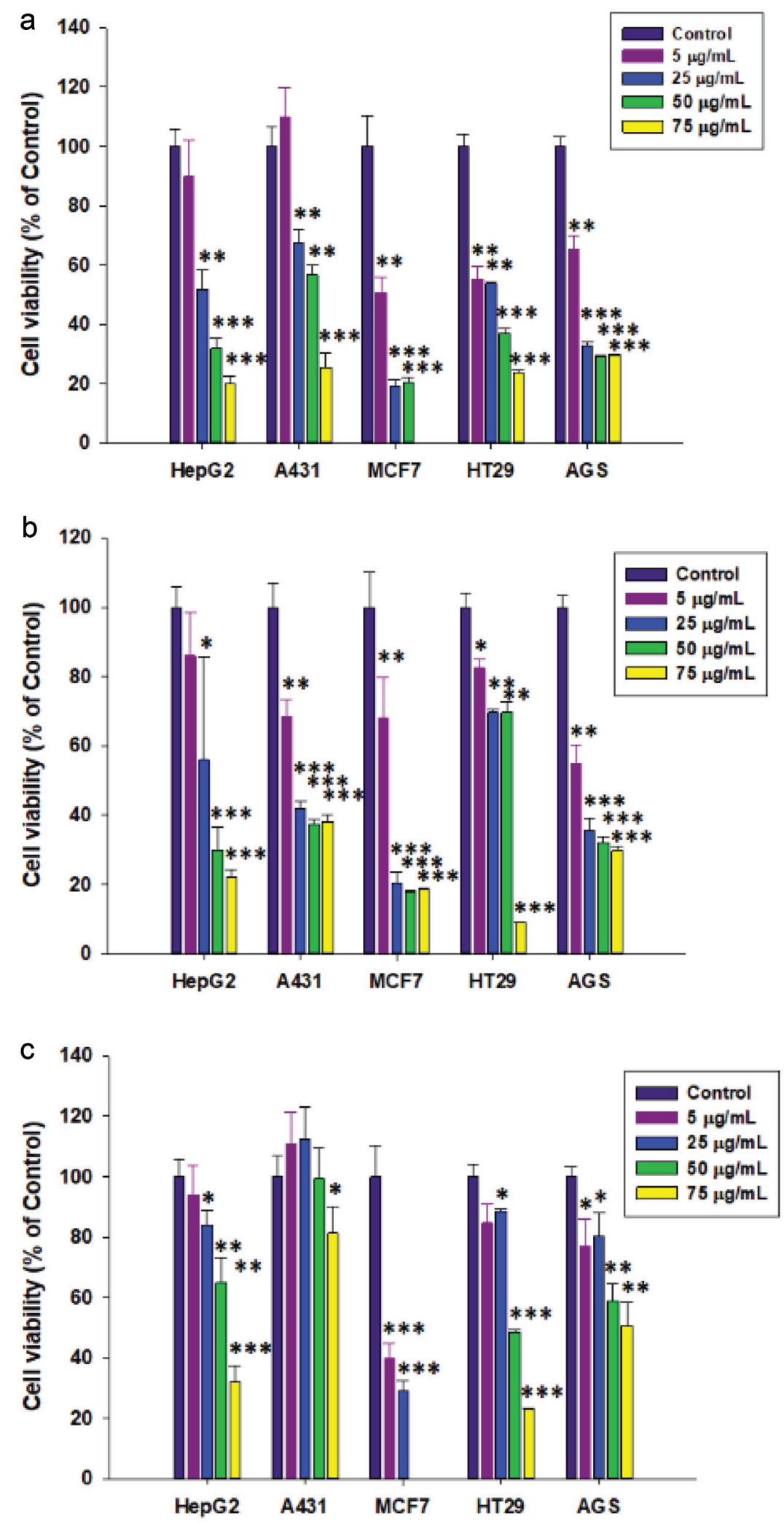

Figure 3. Effect of (a) resveratrol, (b) RC3:0, and (c) RDHA on cell viability of various human cancer cells. Cancer cell lines were treated with different concentrations of resveratrol, RDHA and RC3:0 for $24 \mathrm{~h}$ and then analysed by MTT assay. Results were statistically analysed with one-way ANOVA followed by Tukey's post hoc test. Statistical significance, ${ }^{*} P<0.05,{ }^{* *} P<0.01$ and ${ }^{* * *} P<0.001$, compared with the control group. 
bioavailability might not be possible due to toxicity. Therefore, lipophilised resveratrols need to be evaluated further in vivo for their anti-proliferative effect, which may ultimately lead to anticancer potential.

\section{Summary}

Two resveratrol esters demonstrated anti-inflammatory and antiproliferative activities in this study. The test compounds were able to inhibit NO production in murine RAW 264.7 cells, suggesting their potential anti-inflammatory effect, which needs to be further investigated on the generation of iNOS protein, iNOS gene expression, the release of IL- 6 and TNF- $\alpha$. In addition, all test compounds in this study showed decreased cell viability of HepG2, A431, MCF7, HT-29, and AGS. The results suggest that resveratrol esters need to be further investigated for their anti-inflammatory and anti-proliferative potential.

\section{Acknowledgments}

One of us (FS) thanks the Natural Science and Engineering Research Council (NSERC) of Canada for partial financial support.

\section{References}

Baur, J.A., and Sinclair, D.A. (2006). Therapeutic potential of resveratrol: The in vivo evidence. Nat. Rev. Drug Discov. 5: 493-506.

Bi, X.L., Yang, J.Y., Dong, Y.X., Wang, J.M., Cui, Y.H., Ikeshima, T., Zhao, Y.Q., and Wu, C.F. (2005). Resveratrol inhibits nitric oxide and TNF- $\alpha$ production by lipopolysaccharide-activated microglia. Int. Immunopharmacol. 5: 185-193.

Bove, K., Lincoln, D.W., and Tsan, M.-F. (2002). Effect of resveratrol on growth of 4T1 breast cancer cells in vitro and in vivo. Biochem. Biophys. Res. Commun. 291: 1001-1005.

Cardile, V., Lombardo, L., Spatafora, C., and Tringali, C. (2005). Chemoenzymatic synthesis and cell-growth inhibition activity of resveratrol analogues. Bioorg. Chem. 33: 22-33.

Cichocki, M., Paluszczak, J., Szaefer, H., Piechowiak, A., Rimando, A.M., and Baer-Dubowska, W. (2008). Pterostilbene is equally potent as resveratrol in inhibiting 12-O-tetradecanoylphorbol-13-acetate activated NFKB, AP-1, COX-2, and iNOS in mouse epidermis. Mol. Nutr. Food Res. 52: 62-70.

Coleman, J.W. (2001). Nitric oxide in immunity and inflammation. Int. Immunopharmacol. 1: 1397-1406.

Dercks, W., and Creasy, L.L. (1989). Influence of fosetyl-A1 on phytoalexin accumulation in the Plasmopara viticola-grapevine interaction. Physiol. Mol. Plant Pathol. 34: 203-213.

Donnelly, L.E., Newton, R., Kennedy, G.E., Fenwick, P.S., Leung, R.H.F., Ito, K., Russell, R.E.K., and Barnes, P.J. (2004). Anti-inflammatory effects of resveratrol in lung epithelial cells: Molecular mechanisms. Am. J. Physiol. Lung Cell Mol. Physiol. 287: 774-783.
Fragopoulou, E., Nomikos, T., Karantonis, H.C., Apostolakis, C., Pliakis, E., Samiotaki, M., Panayotou, G., and Antonopoulou, S. (2007). Biological activity of acetylated phenolic compounds. J. Agric. Food Chem. 55: 80-89.

Fransen, M., Nordgren, M., Wang, B., and Apanasets, O. (2012). Role of peroxisomes in ROS/RNS-metabolism: Implications for human disease. Biochim. Biophys. Acta. 1822: 1363-1373.

Halliwell, B. (1996). Free radicals, proteins and DNA: Oxidative damage versus redox regulation. Biochem. Soc. Trans. 24: 1023-1027.

Jang, M., Cai, L., Udeani, G.O., Slowing, K.V., Thomas, C.F., Beecher, C.W.W., Fong, H.H.S., Farnsworth, N.R., Kinghorn, A.D., Mehta, R.G., Moon, R.C., and Pezzuto, J.M. (1997). Cancer chemopreventive activity of resveratrol, a natural product derived from grapes. Science 275 : 218-220.

Kruidenier, L., and Verspaget, H.W. (2002). Review article: Oxidative stress as a pathogenic factor in inflammatory bowel disease - radicals or ridiculous? Aliment. Pharmacol. Ther. 16: 1997-2015.

Loft, S., and Poulsen, H.E. (1996). Cancer risk and oxidative DNA damage in man. J. Mol. Med. 74: 297-312.

Mosmann, T. (1983). Rapid colorimetric assay for cellular growth and survival: Application to proliferation and cytotoxicity assays. J. Immunol. Methods. 65: 55-63.

Oh, W.Y., and Shahidi, F. (2017). Lipophilization of resveratrol and effects on antioxidant activities. J. Agric. Food Chem. 65: 8617-8625.

Pan, M.-H., Chang, Y.-H., Badmaev, V., Nagabhushanam, K., and Ho, C.-T. (2007). Pterostilbene induces apoptosis and cell cycle arrest in human gastric carcinoma cells. J. Agric. Food Chem. 55: 7777-7785.

Patel, K.R., Brown, V.A., Jones, D.J.L., Britton, R.G., Hemingway, D., Miller, A.S., West, K.P., Booth, T.D., Perloff, M., Crowell, J.A., Brenner, D.E., Steward, W.P., Gescher, A.J., and Brown, K. (2010). Clinical pharmacology of resveratrol and its metabolites in colorectal cancer patients. Cancer Res. 70: 7392-7399.

Torres, P., Poveda, A., Jimenez-Barbero, J., Ballesteros, A., and Plou, F.J. (2010). Regioselective lipase-catalyzed synthesis of 3-O-acyl derivatives of resveratrol and study of their antioxidant properties. J. Agric. Food Chem. 58: 807-813.

Tsai, S.-H., Lin-Shiau, S.-Y., and Lin, J.-K. (1999). Suppression of nitric oxide synthase and the down-regulation of the activation of $\mathrm{NFkB}$ in macrophages by resveratrol. Br. J. Pharmacol. 126: 673-680.

Walle, T., Hsieh, F., Delegge, M.H., Oatis, J.E., and Walle, U.K. (2004). High absortion but very low bioavaibility of oral resveratrol in humans. Drug Metab. Dispos. 32(12): 1377-1382.

Wang, Y., Catana, F., Yang, Y., Roderick, R., and van Breemen, R.B. (2002). An LC-MS method for analyzing total resveratrol in grape juice, cranberry juice, and in wine. J. Agric. Food Chem. 50: 431-435.

Wiseman, H., and Halliwell, B. (1996). Damage to DNA by reactive oxygen and nitrogen species: Role in inflammatory disease and progression to cancer. Biochem. J. 313: 17-29.

Yu, B.P. (1994). Cellular defenses against damage from reactive oxygen species. Physiol. Rev. 74: 139-162.

Zhong, M., Cheng, G.F., Wang, W.J., Guo, Y., Zhu, X.Y., and Zhang, J.T. (1999). Inhibitory effect of resveratrol on interleukin 6 release by stimulated peritoneal macrophages of mice. Phytomedicine. 6: 79-84.

Zhong, Y., Chiou, Y.-S., Pan, M.-H., and Shahidi, F. (2012). Anti-inflammatory activity of lipophilic epigallocatechin gallate (EGCG) derivatives in LPS-stimulated murine macrophages. Food Chem. 134: 742-748.

Zhong, Y., and Shahidi, F. (2011). Lipophilized epigallocatechin gallate (EGCG) eerivatives as novel antioxidants. J. Agric. Food Chem. 59: 6526-6533. 